\title{
Recherche clinique et SHES dans le Cancéropôle PACA
}

Christian Chabannon ${ }^{1}$ Roch Giorgi $^{2}$

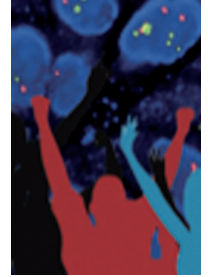

et (2) transférer les connaissances

Dès sa création, le cancéropôle PACA a identifié un axe centré sur les sciences humaines économiques et sociales (SHES) et a affiché clairement qu'il considérait ces disciplines comme des acteurs à part entière de la lutte contre le cancer. L'intégration précoce de ces problématiques dans le périmètre du cancéropôle s'est appuyée sur l'existence dans la région PACA d'un certain nombre d'équipes de recherche développant depuis de nombreuses années des travaux en SHES, travaux menés en collaboration avec des équipes de recherche biomédicale et clinique travaillant dans le champ de l'oncologie. Progressivement, les efforts de structuration et d'animation scientifique ont permis d'attirer vers la problématique du cancer de nouvelles équipes de SHES, d'épidémiologie et de santé publique, et de développer de nouvelles collaborations. La stratégie alors mise en place avait plusieurs buts : (1) améliorer et étendre les synergies préexistantes dans le domaine des cancers entre les équipes régionales des disciplines impliquées dans cet axe du cancéropôle PACA, en collaboration avec les structures de recherche biomédicale et clinique, issues de ces recherches aux équipes biomédicales et cliniques.

Les travaux présentés dans ce numéro témoignent de l'apport de disciplines d'épidémiologie et de SHES, via différentes approches utilisant des méthodes qualitatives et quantitatives autour d'un objet de recherche commun : le développement de la génomique et son transfert à la pratique clinique. En binômes ou trinômes, ces disciplines ont travaillé de manière étroite en associant de façon variée chercheurs, cliniciens ou biologistes, afin de dégager les enjeux de recherche. Ainsi, plusieurs approches complémentaires, génomique, clinique, psycho-épidémiologique, psycho-socio-économique, anthropologique, ou autres combinaisons disciplinaires, ont été mobilisées. L'ensemble de ces travaux illustre le succès d'un des objectifs du cancéropôle PACA : établir des collaborations entre des équipes des domaines SHES, biomédical et clinique et permettre un transfert des connaissances entre la recherche en SHES et la recherche clinique. $\diamond$

Clinical research and human and social sciences at Cancéropôle PACA

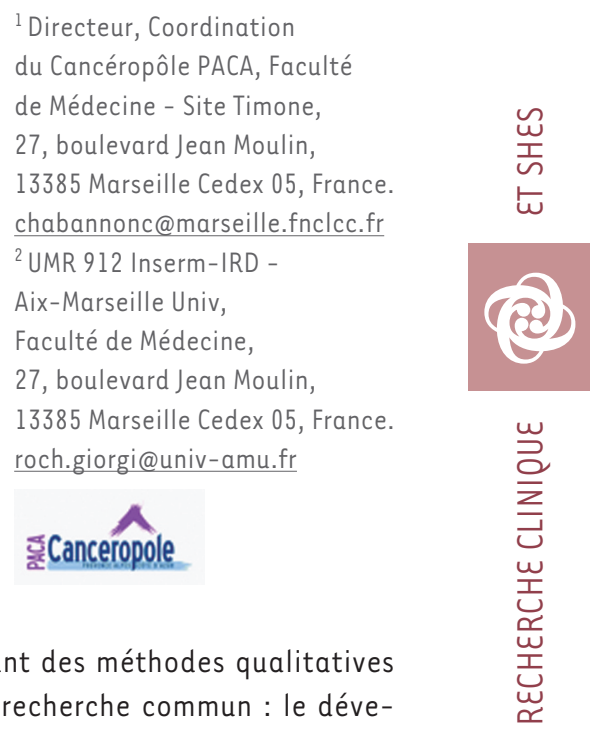
.

.

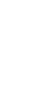

